\title{
Antipsicóticos inyectables de larga duración
}

\section{Long-acting injectable antipsychotics}

Miquel Bioque ${ }^{1}$, Clemente García-Rizo ${ }^{1 *}$ y Marina Garriga²

${ }^{1}$ Unidad de Esquizofrenia, Instituto de Neurociencias, Hospital Clínic, IDIBAPS, CIBERSAM, Universidad de Barcelona; ${ }^{2}$ Unidad de Trastorno Bipolar y Depresivos, Instituto de Neurociencias, Hospital Clínic, CIBERSAM, Barcelona, España

\section{Resumen}

Los antipsicóticos inyectables de larga duración supusieron un cambio de paradigma en el tratamiento de los trastornos psicóticos con reticencias iniciales de terapeutas, pacientes y familiares que perduran hasta la actualidad. La efectividad en la prevención de recaídas asociada a un perfil de efectos secundarios más tolerable que las formulaciones orales ha permitido ampliar las recomendaciones terapéuticas. La retirada de tratamientos de primera generación en los últimos ẫ̃os junto con nuevas formulaciones de segunda generación permitirá una variabilidad terapéutica más acorde con las presćripciones orales. Un conocimiento más exhaustivo de las características de los inyectables de larga duración en nuestro entorno por parte de prescriptores y otros terapeutas, así como mayor difusión a familiares y pacientes, permitirá a estos últimos beneficiarse de las potenciales ventajas del tratamiento antipsicótico inyectable de larga duración.

Palabras claves: Esquizofrenia. Antipsicóticos. Inyectables de larga duración. Recaídas.

\section{Abstract}

Long-acting injectable antipsychotics meant a paradigm shift in the treatment of psychotic disorders with initial reticence that persists until today (attitudes of therapists, patients and family members). The effectiveness in relapse prevention associated with a more tolerable profile of side effects than oral formulations has allowed the expansion of the therapeutic recommendations. The withdrawal of first generation treatments in recent years together with new second generation formulations will allow a therapeutic variability in line with current oral prescriptions. A deeper knowledge of the characteristies of long-term injectables in our environment by prescribers and other therapists as well as greater dissemination of information to family members and patients will allow the latter to take advantage from the potential benefits of long-acting injectable antipsychotic treatment.

Key words: Schizophrenia. Antipsychotics. Long-acting injectable. Psychotic relapse.

Correspondencia:

${ }^{*}$ Clemente Garcia-Rizo

E-mail: cgarcia3@clinic.cat
Fecha de recepción: 09-09-2019

Fecha de aceptación: 26-09-2019

DOI: 10.24875/RPSIC.M19000001
Disponible en internet: 09-12-2019

Revis Psicofarmacol. 2019;5( $(\overline{\mathfrak{P}}: 3-8$ www.revisionesenpsicofarmacologia.com 1138-7165/@ 2019 P. Permanyer. Publicado por Permanyer. Éste es un artículo open access bajo la licencia CC BY-NC-ND (http://creativecommons.org/ licenses/by-nc-nd/4.0/). 


\section{Introducción}

Los antipsicóticos son base del tratamiento para los pacientes con síntomas psicóticos principalmente del espectro de la esquizofrenia ${ }^{1}$. Sin embargo, la falta de adherencia al tratamiento antipsicótico tiene un gran impacto en el curso de la enfermedad, ya que afecta al riesgo de recaída, hospitalización e intentos de suicidio ${ }^{2}$. Un enfoque orientado hacia la recuperación, centrado no solo en la prevención de la recaídas sino también en el funcionamiento, implica las diferentes actitudes de los pacientes y cuidadores con respecto a los tratamientos farmacológicos y no farmacológicos ${ }^{3} \mathrm{e}$ involucra a los diferentes profesionales y las estrategias de tratamiento ${ }^{4}$. Comprender las actitudes de los pacientes y los cuidadores puede ayudar en la adaptación del tratamiento, así como mejorar la alianza terapéutica y la adherencia a la medicación, lo que mejora el pronóstico a largo plazo 5 .

Con frecuencia se considera que las formulaciones inyectables de acción prolongada (ILD) son preferibles a las formas orales, mejoran la adherencia al tratamiento y la prevención de recaídas ${ }^{6}$, a la vez que amplían el número de opciones disponibles para personalizar el tratamiento farmacológico ${ }^{7}$. No solo los antipsicóticos de primera generación (FGA, del inglés first generation antipsychotics), sino varios antipsicóticos de segunda generación (SGA, del inglés second generation antipsychotics) están disponibles como ILD ${ }^{1}$. Los ILD de primera y segunda generación se han estudiado ampliamente en pacientes con trastornos del espectro de la esquizofrenia ${ }^{8,9}$, incluso dos ILD de segunda generación (microesferas de risperidona, como monoterapia o adyuvante a un estabilizador del humor) y el monohidrato de aripiprazol (en monoterapia) han sido aprobados por la Agencia Europea del Medicamento (EMA) y la US Food and Drug Administration (FDA) para el tratamiento de mantenimiento del trastorno bipolar ${ }^{10}$. En nuestro entorno, el uso de ILD se sitúa alrededor del $15 \%$, principalmente SGA, con recomendaciones terapéuticas que abarcan desde la unidad de agudos, niños y adolescentes, hasta primeros episodios psicóticos ${ }^{11}$. En la actualidad incluso se recomienda su uso al menos cuatro meses antes de iniciar tratamiento con clozapina por psicosis refractaria al tratamiento farmacológico ${ }^{12}$.

Complementar el uso de los ILD con los objetivos de los cuidadores y de los pacientes puede contribuir significativamente a lograr su remisión y recuperación?.

\section{Antipsicóticos inyectables de larga duración de primera generación}

Los FGA ILD aparecen en un contexto históricờdeterminado; tras la revolución que supone introducción de la clorpromazina en 1952 para el tratamiento dè-los síntomas psicóticos, la aparición en 1966 del enantato de flufenazina y en 1968 del decanoato de flufenzina fueron vistas con recelo por muchos psiquiatras ${ }^{1 \frac{15}{3}} \mathrm{El}$ concepto de psiquiatría comunitaria, las dudas sobbre el tratamiento continuado, los posibles efectos seeundarios, así como las dudas sobre la imposición del tratamiento que vulneraría la libertad del paciente, generaron mucha controversia en un momento dondê no existían datos sobre las tasas de recaídas y las consecuencias neurobioquímicas y psicosociales de ${ }^{\circ} e s-$ tas. En España, de estos ILD inicialmente conocídos como antipsicóticos depot (de depósito en tejido cêlular subcutáneo) se comercializaron cuatro, dos de, los cuales ya no están en el mercado (Tabla 1): palmitato de pipotiazina $25 \mathrm{mg} / \mathrm{ml}$ (Lonseren $\left.{ }^{\circledR}, 1973-2015\right)$, dèc canoato de flufenazina $25 \mathrm{mg} / \mathrm{ml}$ (Modecate ${ }^{\circledR}, 1974-2019$ ), decanoato de zuclopentixol $200 \mathrm{mg} / \mathrm{ml}$ (Clopixol'De$\left.\operatorname{pot}^{\circledR}, 1995-\right)^{14}$ y acetato de zuclopentixol $50 \mathrm{mg} / \mathrm{ml}$ (Clopixol Acufase ${ }^{\circledR} 1995-$; hasta 2005 se comerciälizó también bajo el nombre de Cisordinol Acufase $\left.{ }^{\circledR}\right)^{15} \mathrm{~g}$ zuclopentixol (isómero cis clopentixol) introducidōen 1978 tiene afinidad por receptores dopaminérgicos D1 y D2, adrenorreceptores $\alpha 1$ y serotoninérgicos 5 갠 sin afinidad por los colinérgicos muscarínicos.

\section{Antipsicóticos inyectables de segunda generación}

La aparición en 2003 del ILD de risperidona (ef̣primer SGA aprobado en 1993 por la FDA) suponę un cambio de modelo en el uso de ILD al poder incluíslos beneficios de los SGA.

\section{Risperidona inyectable de larga duración}

La risperidona bloquea receptores cerebrales de dopamina D2 y, en mayor proporción, receptores $5 \mathrm{HT}_{2}$ de serotonina ${ }^{16}$. En un ensayo clínico aleatorizado (ECA) de 12 semanas de duración que incluyó 554 pacientes con esquizofrenia crónica, la risperidona injectable de larga duración (RILD) demostró por primera vez ser eficaz y bien tolerada en comparación con placebo ${ }^{17}$, y especialmente útil para pacientes que presentan dificultades para adherirse al tratamiento oral ${ }^{6}$. Posteriormente, en un ECA con 86 participantes, el uso 
Tabla 1. Antipsicóticos inyectables de larga duración de primera generación

\begin{tabular}{|c|c|c|c|c|c|}
\hline & $\begin{array}{l}\text { Palmitato de } \\
\text { pipotiazina } \\
\text { (Lonseren }{ }^{\circledR} \text { ) }\end{array}$ & $\begin{array}{l}\text { Decanoato de flufenazina } \\
\text { (Modecate }{ }^{\circledR} \text { ) }\end{array}$ & $\begin{array}{l}\text { Decanoato de } \\
\text { zuclopentixol } \\
\text { (Clopixol Depot }^{\circledR} \text { ) }\end{array}$ & \multicolumn{2}{|c|}{$\begin{array}{l}\text { Acetato de } \\
\text { zuclopentixol* } \\
\text { (Clopixol Acufase }{ }^{\circledR} \text { ) }\end{array}$} \\
\hline Formulación & Oleosa & Oleosa & Oleosa & Oleosa & हृ \\
\hline Dosis habitual & $100 \mathrm{mg}$ & $12,5-25 \mathrm{mg}$ & $200-400 \mathrm{mg}$ & $50-150 \mathrm{mg}$ & $\frac{\bar{\alpha}}{2}$ \\
\hline Frecuencia dosis & 4 semanas & 4-6 semanas & 2-4 semanas & 2-3 días & \\
\hline $\begin{array}{l}\text { Lugar de } \\
\text { administración }\end{array}$ & \multicolumn{2}{|c|}{ La ficha técnica no lo especifica, aunque suele ser glúteo } & Glúteo & Glúteo & :음 \\
\hline & & & \multicolumn{2}{|c|}{$\begin{array}{l}\text { Ambos pueden mezclarse en una jeringa } \\
\text { administrarse como una sola } \\
\text { inyección (coinyección) }\end{array}$} & $y \frac{\bar{c}}{\frac{\bar{a}}{\bar{c}}}$ \\
\hline & & & \multicolumn{2}{|c|}{$\begin{array}{l}\text { Volúmenes de inyección que excedan de } \\
\text { deben distribuirse entre dos lugares de } \\
\text { inyección }\end{array}$} & 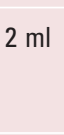 \\
\hline & $\begin{array}{l}\text { https://cima.aemps. } \\
\text { es/cima/pdfs/es/ } \\
\text { ft/51469/FT_51469.pdf }\end{array}$ & $\begin{array}{l}\text { https://cima.aemps.es/cima/pdfs/es/ } \\
\text { ft/52602/FichaTecnica_52602.html.pdf }\end{array}$ & \multicolumn{2}{|c|}{ https://cima.aemps.es/cima/publico/home } & 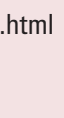 \\
\hline
\end{tabular}

*Se recomienda una duración del tratamiento no mayor de 2 semanas, una dosis máxima acumulada durante un curso de tratamiento no mayor de 400 mg y un númêro de inyecciones no superior a 4 .

Tabla 2. Formulaciones de aripiprazol inyectable de larga duración

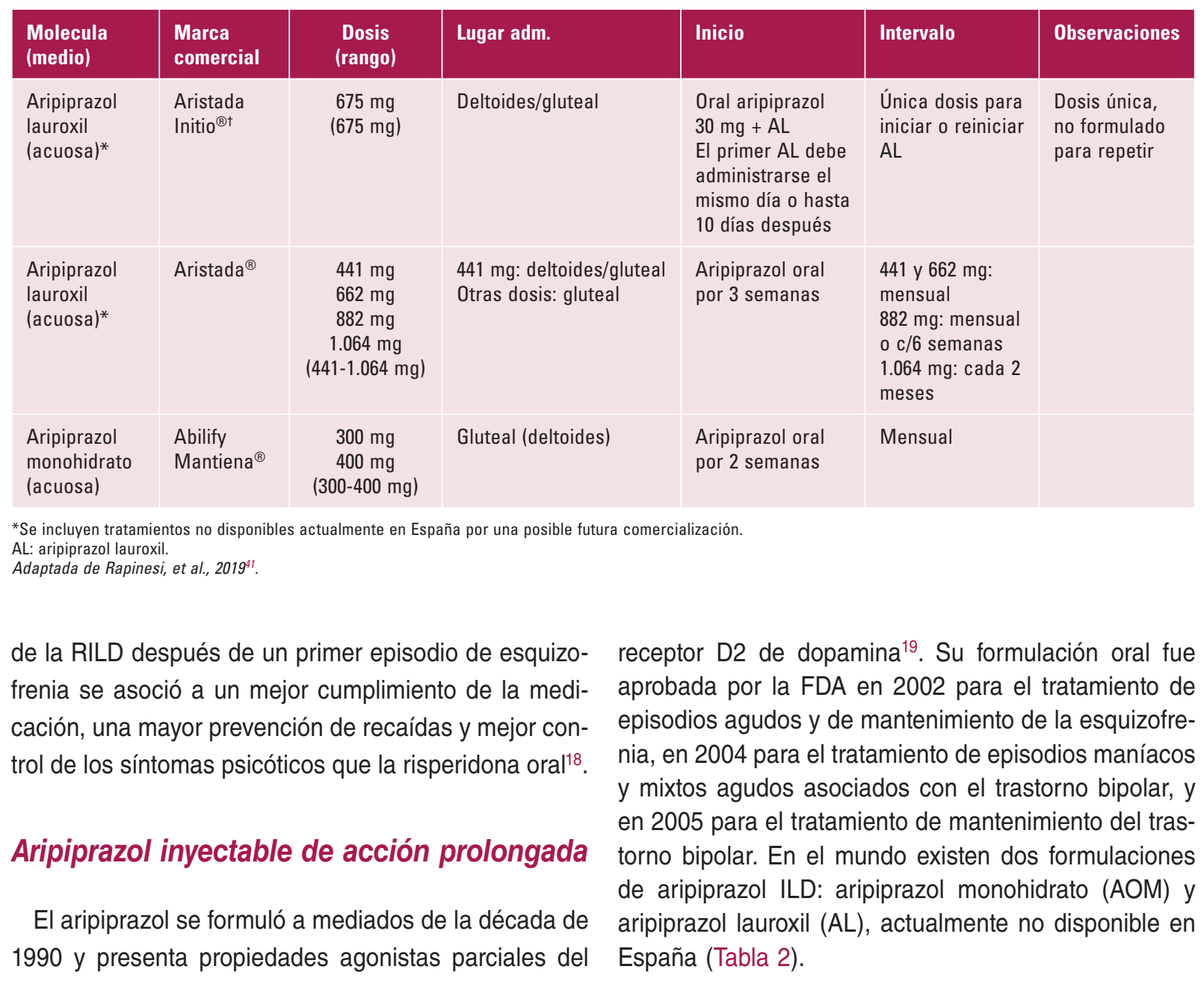


Tabla 3. Comparativa entre los diferentes antipsicóticos inyectables de acción prolongada disponibles

\begin{tabular}{|c|c|c|c|c|c|c|}
\hline & $\begin{array}{l}\text { Palmitato de } \\
\text { paliperidona } \\
\text { trimestral }\end{array}$ & $\begin{array}{l}\text { Aripiprazol } \\
\text { monohidrato }\end{array}$ & $\begin{array}{l}\text { Palmitato de } \\
\text { paliperidona } \\
\text { mensual }\end{array}$ & $\begin{array}{l}\text { Pamoato de } \\
\text { olanzapina }\end{array}$ & $\begin{array}{l}\text { Risperidona } \\
\text { inyectable } \\
\text { larga } \\
\text { duración }\end{array}$ & $\begin{array}{l}\text { Zuclopent } \\
\text { decanoatc }\end{array}$ \\
\hline Formulación & Acuosa & Acuosa & Acuosa & Acuosa & Acuosa & Oleosa \\
\hline $\begin{array}{l}\text { Necesidad de suplementación } \\
\text { oral al inicio del tratamiento }\end{array}$ & No & Sí & No & Variable & Sí & Si \\
\hline Días entre administraciones & 90 & 30 & 30 & $15-30$ & 15 & $200-400$ \\
\hline Lugar de administración & $\begin{array}{l}\text { Glúteo y } \\
\text { deltoides }\end{array}$ & $\begin{array}{l}\text { Glúteo y } \\
\text { deltoides }\end{array}$ & $\begin{array}{l}\text { Glúteo y } \\
\text { deltoides }\end{array}$ & Glúteo & $\begin{array}{l}\text { Glúteo y } \\
\text { deltoides }\end{array}$ & Glúteo \\
\hline $\begin{array}{l}\text { Dosis disponibles } \\
\text { (en } \mathrm{mg} \text { ) }\end{array}$ & $\begin{array}{l}175,263,350 y \\
525\end{array}$ & 300 y 400 & $\begin{array}{l}50,75,100 y \\
150\end{array}$ & 210,300 y 405 & $25,37,5$ y 50 & 200 \\
\hline Necesidad de reconstrucción & $\begin{array}{l}\text { No (jeringas } \\
\text { precargadas) }\end{array}$ & Sí & $\begin{array}{l}\text { No (jeringas } \\
\text { precargadas) }\end{array}$ & Sí & Sí & No \\
\hline Necesidad de refrigeración & No & No & No & No & Sí & No \\
\hline $\begin{array}{l}\text { Necesidad de vigilancia por } \\
\text { síndrome postinyección }\end{array}$ & No & No & No & Sí (3 horas) & No & No \\
\hline
\end{tabular}

No se incluye acetato de zuclopentixol por su recomendación limitada de tratamiento temporal (Tabla 1). Adaptada de Bernardo, et al., $2016^{42}$.

El AOM y el AL comparten características comunes basadas en la propia molécula (aripiprazol), pero difieren en términos de farmacocinética e instrucciones de uso. Las concentraciones plasmáticas máximas se alcanzan a los 5-7 días, de ahí la necesidad de una suplementación oral inicial de al menos 14 días para asegurar concentraciones terapéuticas ${ }^{20}$.

Ambas formulaciones (AL y AOM) han mostrado ser eficaces en el tratamiento de todas las fases de la esquizofrenia y en la prevención de recaídas. En la exacerbación aguda de la esquizofrenia, un ECA de AOM $(400 \mathrm{mg})^{21}$ y AL (441 y $\left.882 \mathrm{mg}\right)^{22}$ mostró una reducción temprana sintomatológica en comparación con el placebo. También se observó un perfil de seguridad y tolerabilidad aceptable, con diminución de los niveles de prolactina ${ }^{22}$. En el tratamiento de mantenimiento de la esquizofrenia, AOM retrasó significativamente el tiempo de recaída ${ }^{23} y$ fue bien tolerada como tratamiento de mantenimiento en comparación con el placebo. Respecto al trastorno bipolar, solo hay un estudio internacional multicéntrico del uso de AOM para el trastorno bipolar I; este ECA de 52 semanas de seguimiento después de una fase maniaca mostró como el AOM 400 mg retrasaba el tiempo de recurrencia en comparación con el placebo, siendo seguro y bien tolerado ${ }^{24}$.

\section{Olanzapina pamoato}

La formulación de pamoato de olanzapina (OP) fue aprobada por la FDA en 2009 para el tratamiento de la esquizofrenia ${ }^{25}$. Sin embargo, preocupaciones en torno a su administración y el riesgo de sufrir el llamado «síndrōme postinyección» impusieron en su administración un pẹriodo de supervisión hospitalario de 3 horas tras su administración. Los síntomas del «síndrome postinyección» pueden incluir sedación intensa, delirio, ataxia, confusión u otras alteraciones del estado de consciencia. Sin embargo, su relación causal sigue sin estar clara, sugifiéndose que una cantidad excesiva de olanzapina pueda haber llegado a la circulación sistémica después de üna inyección intravascular inadvertida ${ }^{26}$ (Tabla 3).

Hasta la fecha, no se ha publicado ningún ECA en pacientes con trastorno bipolar ${ }^{27}$, pero sí en pacientes con esquizofrenia. En el tratamiento agudo de la esquizofrenia, el OP $(210 \mathrm{mg} / 2 \mathrm{sem}, 300 \mathrm{mg} / 2 \mathrm{sem}$ y $405 \mathrm{mg} / 4 \mathrm{sem})$ demostró ser tan eficaz como suchomólogo oral (olanzapina 2,5-15 mg/d) y superior a palacebo en remisión clínica y tasas de discontinuaciôn ${ }^{28}$. En cuanto al tratamiento de mantenimiento, el OP $\$ 50$, $300,405 \mathrm{mg}$ fue superior a $45 \mathrm{mg}$ en el tiempo fibre de enfermedad hasta la siguiente recaída y en la puesta clínica mantenida ${ }^{29}$. Las diferentes dosis de $\mathrm{OP}$ han mostrado ser tan efectivas como sus homólogos orales en el mantenimiento de la respuesta clínica ${ }^{30}$.

\section{Palmitato de paliperidona mensual}

La paliperidona es el metabolito activo de la risperidona (9-OH-risperidona) y bloquea receptores $5-\mathrm{HT}_{2 \mathrm{~A}}$, D2, adrenoceptores alfa 1 y alfa 2 , y receptores de la 
histamina 1. A diferencia de la risperidona, la paliperidona no bloquea los adrenoceptores beta o los receptores muscarínicos, y muestra también una menor afinidad por los receptores D4 ${ }^{31}$ (Tabla 3).

El palmitato de paliperidona mensual (PP1M) fue inicialmente aprobado por la FDA en 2009, en un rango de dosis de $25-150 \mathrm{mg}$ al mes $^{31}$. Como régimen de inicio de tratamiento se recomiendan inyecciones en deltoides de $150 \mathrm{mg}$ y de $100 \mathrm{mg}$ el octavo día $( \pm 4)$ con el fin de alcanzar rápidamente concentraciones plasmáticas en rango terapéutico, seguidas por inyecciones mensuales de administración glútea 0 deltoides ${ }^{31}$. La suplementación oral no es necesaria, aunque algunos pacientes podrían beneficiarse ${ }^{32}$. En el año 2015 una revisión de nuestro grupo identificó 18 estudios de PP1M, reportando la superioridad del PP1M respecto a placebo y resultados similares de eficacia y tolerabilidad en comparación con la RILD o la paliperidona oral ${ }^{33}$.

En un reciente estudio multicéntrico, abierto, prospectivo y observacional, el PP1M resultó ser eficaz y bien tolerado para tratar fases agudas de esquizofrenia, tanto solo como combinado con otros antipsicóti$\cos ^{32}$. Por último, una revisión reciente de estudios de real-world data (procedentes de estudios observacionales y ECA pragmáticos) indicó que el PP1M es superior a los antipsicóticos orales en retrasar el fracaso terapéutico o las recaídas ${ }^{34}$.

\section{Palmitato de paliperidona trimestral}

El palmitato de paliperidona trimestral (PP3M) se comercializó en 2015 en los EE.UU. y en 2016 en la Unión Europea y otras partes del mundo, indicado para el tratamiento de la esquizofrenia en pacientes adecuadamente estabilizados con PP1M. Por ficha técnica, una estabilización previa de mínimo de cuatro meses con PP1M era obligatoria en EE.UU. y recomendable en Europa $^{35}$ (Tabla 3).

Los ensayos de simulación demostraron que los niveles plasmáticos de paliperidona tras cambiar a PP3M (175-525 mg/EQ cada 12 semanas) eran similares a los obtenidos con las correspondientes dosis de PP1M (50$150 \mathrm{mg} / \mathrm{EQ}$ cada 4 semanas) o paliperidona oral (4-12 mg, diario $^{36}$. La biodisponibilidad era totalmente independiente del sitio de inyección y de la dosis ${ }^{37}$. No es necesario un ajuste de dosificación según las diferencias de sexo, índice de masa corporal, sitio de la inyección o edad. A diferencia del PP1M, la administración de PP3M puede ser en deltoides o glúteos desde el inicio ${ }^{35}$.

Un primer ECA de 283 pacientes con esquizofrenia demostró la eficacia del PP3M en retrasar el tiempo hasta la recaída en comparación con placebo ${ }^{38}$. En un segundo ECA con 1.016 pacientes el PP3M demostró no ser inferior al PP1M, mostrando una tasa de recaídas similar ( $8 \%$ con PP3M vs. $9 \%$ con PP1M) ${ }^{39}$. Posteriormente Weiden, et al. compararon el efecto de la paliperidona oral, el PP1M y el PP3M en el tiempo hästa la recaída tras la discontinuación, informando que la formulación con la semivida más larga (PP3M) proporciona la mayor prevención de recaídas tras el abanindo$\mathrm{no}^{40}$. Los efectos adversos reportados en los ECA $\mathrm{del}$ PP3M son similares a sus compuestos relacionados (paliperidona y risperidona) y similares al $\mathrm{PP} 1 \mathrm{M}^{37,39}$

\section{Conclusiones}

Los psiquiatras disponemos en la actualidad dé diferentes opciones terapéuticas con inyectables de larga duración, principalmente con SGA (Tabla 3), que permiten ofrecer a paciente y familiares tratamientos contrastados científicamente que, combinados coñ diversas opciones psicoterapéuticas y psicosociąles, ayuden al control de la sintomatología, eviten lasipotenciales recaídas y optimicen la funcionalidad diaria.

\section{Financiación}

Ninguna.

\section{Conflictos de interés}

El Dr. Bioque ha recibido honorarios de difusión medica/consultoría de Adamed, Angelini, Ferrer, Jänssen-Cilag, Lundbeck, Neuraxpharm y Otsuka y ha sido galardonado con un premio de investigación de Pfizer. La Dra. Garriga ha recibido honorarios de difusión mimedica/consultoría de Ferrer, Lundbeck y Janssen-Cilag:así como financiación del Ministerio de Economía y Comp̄etitividad, Instituto de Salud Carlos III, a través de un contrato de 'R1o Hortega' (CM17/00102) y Centro de Investigaeión Biomédica en Red de Salud Mental (CIBERSAM).

El Dr. García-Rizo ha recibido honorarios de difưsión medica/consultoría de Adamed, Alter, Janssen-Cilä̆g y Lundbeck y financiación mediante una beca de inyestigación del Ministerio de Ciencia, Innovación y Unìyersidades e Instituto de Salud Carlos III (PI14/00753).

\section{Responsabilidades éticas}

Protección de personas y animales. Los autores declaran que para esta investigación no se han reađizado experimentos en seres humanos ni en animales. 
Confidencialidad de los datos. Los autores declaran que han seguido los protocolos de su centro de trabajo sobre la publicación de datos de pacientes.

Derecho a la privacidad y consentimiento informado. Los autores declaran que en este artículo no aparecen datos de pacientes.

\section{Bibliografía}

1. Stevens GL, Dawson G, Zummo J. Clinical benefits and impact of early use of long-acting injectable antipsychotics for schizophrenia. Early Interv Psychiatry. 2016;10(5):365-77

2. Leucht S, Heres S. Epidemiology, clinical consequences, and psychosocial treatment of nonadherence in schizophrenia. J Clin Psychiatry. 2006:67(Suppl 5):3-8.

3. American Psychiatric Association. Diagnostic and Statistical Manual of Mental Disorders, Fifth Edition. Washington, DC: American Psychiatric Association Publishing; 2013.

4. Kreyenbuhl J, Buchanan RW, Dickerson FB, Dixon LB; Schizophrenia Patient Outcomes Research Team (PORT). The Schizophrenia Patien Outcomes Research Team (PORT): Updated treatment recommendations 2009. Schizophr Bull. 2010;36(1):94-103.

5. Naber D. Subjective effects of antipsychotic treatment. Acta Psychiatr Scand. 2005;111(2):81-3.

6. Leucht C, Heres S, Kane JM, Kissling W, Davis JM, Leucht S. Oral versus depot antipsychotic drugs for schizophrenia--a critical systematic review and meta-analysis of randomised long-term trials. Schizophr Res. 2011;127(1-3):83-92.

7. Altamura AC, Aguglia E, Bassi M, Bogetto F, Cappellari L, De Giorgi S, et al. (2012). Rethinking the role of long-acting atypical antipsychotics in the community setting. Int Clin Psychopharmacol. 2012;27(6):336-49.

8. Kishimoto T, Nitta M, Borenstein M, Kane JM, Correll CU. Long-acting injectable versus oral antipsychotics in schizophrenia: a systematic review and meta-analysis of mirror-image studies. J Clin Psychiatry. 2013;74(10):957-65

9. Misawa F, Kishimoto T, Hagi K, Kane JM, Correll CU. Safety and tolerability of long-acting injectable versus oral antipsychotics: A meta-analysis of randomized controlled studies comparing the same antipsychotics. Schizophr Res. 2016;176(2-3):220-30.

10. Keramatian K, Chakrabarty T, Yatham LN. Long-acting injectable second-generation/atypical antipsychotics for the management of bipolar disorder: a systematic review. CNS Drugs. 2019;33(5):431-56.

11. Arango C, Baeza I, Bernardo M, Cañas F, de Dios C, Díaz-Marsá M, et al. Antipsicóticos inyectables de I+iberación prolongada para el tratamiento de la esquizofrenia en España. Rev Psiquiatr Salud Ment (Barc.). 2019;12(2):92-105.

12. Howes OD, McCutcheon R, Agid O, De Bartolomeis A, van Beveren NJM, Birnbaum ML, et al. Treatment-resistant schizophrenia: Treatment Response and Resistance in Psychosis (TRRIP) Working Group Consensus Guidelines on Diagnosis and Terminology. Am J Psychiatry. 2017;174(3):216-29.

13. Johnson DA. Historical perspective on antipsychotic long-acting injections. Br J Psychiatry Suppl. 2009:52:S7-12.

14. da Silva Freire Coutinho E, Fenton M, Quraishi SN. Zuclopenthixol decanoate for schizophrenia and other serious mental illnesses. Cochrane Database Syst Rev. 2000;(2):CD001164.

15. Gibson RC, Fenton M, da Silva Freire Coutinho E, Campbell C. Zuclopenthixol acetate for acute schizophrenia and similar serious mental illnesses. Cochrane Database of Systematic Reviews. https://doi. org/10.1002/14651858.cd000525.pub2

16. Sampson S, Hosalli P, Furtado VA, Davis JM. Risperidone (depot) for schizophrenia. Cochrane Database Syst Rev. 2016 Apr 14;4:CD004161.

17. Kane JM, Eerdekens M, Lindenmayer JP, Keith SJ, Lesem M, Karcher K Long-acting injectable risperidone: Efficacy and safety of the first long-acting atypical antipsychotic. Am J Psychiatry. 2003;160(6):1125-32.

18. Subotnik KL, Casaus LR, Ventura J, Luo JS, Hellemann GS Gretchen-Doorly D, et al. Long-acting injectable risperidone for relapse prevention and control of breakthrough symptoms after a recent first episode of schizophrenia a randomized clinical trial. JAMA Psychiatry. 2015;72(8):822-9.

19. Fujikawa M, Nagashima M, Inoue T, Yamada K, Furukawa T. Partial agonistic effects of OPC-14597, a potential antipsychotic agent, on yawning behavior in rats. Pharmacol Biochem Behav. 1996;53(4):903-9

20. Mallikaarjun S, Kane JM, Bricmont P, McQuade R, Carson W, Sanchez R, et al. Pharmacokinetics, tolerability and safety of aripiprazole once-monthly in adult schizophrenia: an open-label, parallel-arm, multiple-dose study. Schizophr Res. 2013;150(1):281-8.

21. Ismail Z, Peters-Strickland T, Miguelez M, Baker RA, Hertel P, Eramo A, et al. Aripiprazole once-monthly in the treatment of acute psychotic episodes in schizophrenia: post hoc analysis of positive and negative syndrome scale marder factor scores. J Clin Psychopharmacol. 2017;37(3):347-50.
22. Citrome L, Risinger R, Cutler AJ, Du Y, Zummo J, Nasrallah HA, et al. Effect of aripiprazole lauroxil in patients with acute schizophrenia as assessed by the Positive and Negative Syndrome Scale-sup̄̄ortive analyses from a Phase 3 study. CNS Spectr. 2018;23(4):284-90.

23. Kane JM, Sanchez R, Perry PP, Jin N, Johnson BR, Forbes RA et al. Aripiprazole intramuscular depot as maintenance treatment in pattents with schizophrenia: a 52-week, multicenter, randomized, double-blind, placebo-controlled study. J Clin Psychiatry. 2012;73(5):617-24.

24. Calabrese JR, Sanchez R, Jin N, Amatniek J, Cox K, Johnson B, Cet al. Efficacy and safety of aripiprazole once-monthly in the maintenance treatment of bipolar I disorder: a double-blind, placebo-controlled, 52 week randomized withdrawal study. J Clin Psychiatry. 2017;78(3):324-31.

25. Remenar JF. Making the leap from daily oral dosing to long-acting injectables: lessons from the antipsychotics. Mol Pharm. 2014;11(6):1739-49.

26. Naber D. Olanzapine pamoate for the treatment of schizophrenia. Expert Opin Pharmacother. 2011 Mar;12(4):627-33

27. Pacchiarotti I, Tiihonen J, Kotzalidis GD, Verdolini N, Murru A Goikolea JM, et al. Long-acting injectable antipsychotics (LAls) formaintenance treatment of bipolar and schizoaffective disorders: A systematic review. Eur Neuropsychopharmacol. 2019;29(4):457-70.

28. Lauriello J, Lambert T, Andersen S, Lin D, Taylor CC, McDonnell D. An 8-week, double-blind, randomized, placebo-controlled study of olanzapine long-acting injection in acutely ill patients with schizophrenia. J Clify.Psychiatry. 2008;69(5):790-9. Erratum en: J Clin Psychiatry. 2011;72(8):1157.

29. Detke HC, Weiden PJ, Llorca PM, Choukour M, Watson SB, Brunner E, et al. Comparison of olanzapine long-acting injection and oral olanzapine: a 2-year, randomized, open-label study in outpatients with schizophrenia. J Clin Psychopharmacol. 2014;34(4):426-34.

30. Kane JM, Detke HC, Naber D, Sethuraman G, Lin DY, Bergstrom RF, et al. Olanzapine long-acting injection: a 24-week, randomized double-blind trial of maintenance treatment in patients with schizophrenia. Am J Psychiatry. 2010;167(2):181-9.

31. INVEGA SUSTENNA ${ }^{\circledR}$ [Internet]. Janssen Pharmaceutical Compānies; 2009. Disponible en: http://www.janssenlabels.com/package-inseit/product-monograph/prescribing-information/INVEGA+SUSTENNA-pi.pdf

32. Parellada E, Bioque M, Serrano M, Herrera B, García Dorado M, SHADOW Study Group. An open-treatment six-week study of the clinical effectiveness of Paliperidone Palmitate in schizophrenia: data from acute units in Spain (SHADOW study). Int J Psychiatry Clin pract. 2018;22(3):191-9.

33. González-Rodríguez A, Catalán R, Penadés R, García-Rizo C, Bioqūe M, Parellada $\mathrm{E}$, et al. Profile of paliperidone palmitate once-monthly long-acting injectable in the management of schizophrenia: Long-term safety, efficacy, and patient acceptability - A review. Patient Prefer Adherence. 2015:9:695-706

34. Emsley R, Parellada E, Bioque M, Herrera B, Hernando T, García-Dorado M. Real-world data on paliperidone palmitate for the treatment of schizophrenia and other psychotic disorders: A systematic review of randomized and nonrandomized studies. Int Clin Psychopharmacol. 2018;33(1):15-33.

35. Trevicta (previously Paliperidone Janssen) [Internet]. European Mếdicines Agency; 2018. Disponible en: http://www.ema.europa.eu/ema/tindex. jsp?curl=pages/medicines/human/medicines/004066/huflan_ med 001829.jsp\&mid=WC0b01ac058001d124

36. Magnusson MO, Samtani MN, Plan EL, Jonsson EN, Rossenu S, Vermeulen A, et al. Dosing and switching strategies for paliperidone palmitate 3-month formulation in patients with schizophrenia based on population pharmacokinetic modeling and simulation, and clinica trial data. CNS Drugs. 2017;31(4):273-88.

37. Ravenstijn P, Remmerie B, Savitz A, Samtani MN, Nuamah I, Chang CT, et al. Pharmacokinetics, safety, and tolerability of paliperidone palmitate 3-month formulation in patients with schizophrenia: A phase-1, singte-dose, randomized, open-label study. J Clin Pharmacol. 2016;56(3):330-9.

38. Berwaerts J, Liu Y, Gopal S, Nuamah I, Xu H, Savitz A, et al. Efficacy and safety of the 3-month formulation of paliperidone palmitate vs placebo for relapse prevention of schizophrenia a randomized clinicaftrial. JAMA Psychiatry. 2015;72(8):830-9.

39. Savitz AJ, Xu H, Gopal S, Nuamah I, Hough D, Mathews M. Paliperidone palmitate 3-month treatment results in symptomatic remission patients with schizophrenia: A randomized, multicenter, double-blin $\bar{x}$, and noninferiority study. Int Clin Psychopharmacol. 2017;32(6):329-36?

40. Weiden PJ, Kim E, Bermak J, Turkoz I, Gopal S, Berwaerts J.Does half-life matter after antipsychotic discontinuation? A relapse comparison in schizophrenia with 3 different formulations of paliperidone. J Clin-Psychiatry. 2017;78(7):e813-20.

41. Rapinesi C, Kotzalidis GD, Mazzarini L, Brugnoli R, Ferracuti S, De Filippis $\mathrm{S}$, et al. Long-acting injectable ( $\mathrm{LAl}$ ) aripiprazole formulations in the treatment of schizophrenia and bipolar disorder: a systematic review Clin Drug Investig. 2019;39(8):713-35.

42. Bernardo $M$, Bioque $M$. Three-month paliperidone palmitate - A new treatment option for schizophrenia. Expert Rev Clin Pharmacol. 2016;9(7):899-904. 